\title{
A systematic review and meta-analysis: value of ultrasound- guided vacuum-assisted biopsy in the diagnosis and treatment of breast lesions
}

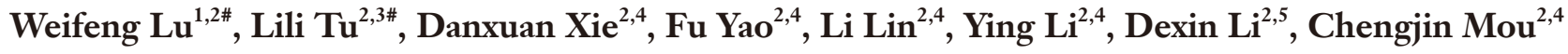 \\ ${ }^{1}$ Department of Anesthesia Surgery Center, Sichuan Provincial People's Hospital, University of Electronic Science and Technology of China, Chengdu, \\ China; ${ }^{2}$ Chinese Academy of Sciences Sichuan Translational Medicine Research Hospital, Chengdu, China; ${ }^{3}$ Department of Geriatric Endocrinology, \\ Sichuan Provincial People's Hospital, University of Electronic Science and Technology of China, Chengdu, China; ${ }^{4}$ Department of Thyroid and \\ Breast Surgery, Sichuan Academy of Medical Sciences \& Sichuan Provincial People's Hospital, Eastern Hospital, Chengdu, China; ${ }^{5}$ Department of \\ Hepatobiliary Surgery, Sichuan Academy of Medical Sciences \& Sichuan Provincial People's Hospital, Eastern Hospital, Chengdu, China \\ Contributions: (I) Conception and design: W Lu, L Tu, D Li, C Mou; (II) Administrative support: D Xie, F Yao; (III) Provision of study materials or \\ patients: W Lu, L Tu, L Lin, Y Li; (IV) Collection and assembly of data: All authors; (V) Data analysis and interpretation: D Xie, F Yao, L Lin, Y Li, \\ D Li, C Mou; (VI) Manuscript writing: All authors; (VII) Final approval of manuscript: All authors. \\ \#These authors contributed equally to this work. \\ Correspondence to: Dexin Li; Chengjin Mou. No.585, Honghe North Road, Chengdu, China. Email: lidexin2188@163.com; mcj2hh@163.com.
}

Background: In recent years, according to global statistics, breast cancer is the main disease affecting women. Ultrasound-guided vacuum-assisted biopsy (VAB) has become a frequently used method for breast cancer detection because of its accuracy, simplicity, and fewer complications.

Methods: In PubMed, Medline, EMbase and Cochrane central register of controlled trials, the retrieval time was from the establishment of the database to March 2021, and the keywords included breast tumor, breast cancer-related diseases, breast lesions, vacuum-assisted breast biopsy, sensitivity and specificity. Metaanalysis was performed using RevMan5.3 software provided by the Cochrane Collaboration.

Results: A total of 10 articles were included using a random-effects model that pooled the sensitivity, specificity, and other accuracy measures of VAB. The summary receiver operating characteristic (SROC) characteristic curve was used to summarize the overall accuracy. The sensitivity range was 0.94 to 1.00 (mean, $0.981 ; 95 \%$ CI, 0.972-0.987) with a specificity range of 0.87-1.00 (mean, 0.999; 95\% CI, 0.997-0.999). The preoperative platelet-lymphocyte ratio (PLR) was 93.84 (95\% CI, 41.55-211.95), the neutrophil to lymphocyte ratio (NLR) was 0.05 (95\% CI, 0.03-0.09), the sensitivity and specificity of $\chi^{2}$ were 37.10 $(\mathrm{P}=0.011)$ and $32.00(\mathrm{P}=0.043)$, respectively, while those of PLR, NLR, and duration of response (DOR) were 46.98 ( $\mathrm{P}=0.001), 54.92$ ( $\mathrm{P}=0.001)$, and 43.49 ( $\mathrm{P}=0.002)$, respectively. Differences were considerable.

Discussion: In this meta-analysis, a total of 10 articles were included. VAB is an accurate type of biopsy to detect female breast cancer. The results of the meta-analysis were stable, and VAB had high sensitivity (98\%) and specificity (nearly 100\%).

Keywords: Breast cancer; vacuum-assisted biopsy (VAB); ultrasonic; meta-analysis

Submitted Aug 12, 2021. Accepted for publication Oct 11, 2021.

doi: $10.21037 / g s-21-611$

View this article at: https://dx.doi.org/10.21037/gs-21-611 


\section{Introduction}

According to the global statistics, breast cancer is the main disease affecting women, with more than two million women diagnosed with breast cancer, and more than 700,000 deaths from breast cancer, which comprises a large proportion of cancer deaths (1). Deaths from breast cancer account for about $20 \%$ of global deaths (2). Early detection, early diagnosis, and early treatment can effectively improve the cure rate and survival rate of breast cancer patients, and accurate diagnosis is crucial for the follow-up treatment of breast cancer patients. The vacuum-assisted biopsy system was introduced in 1995. Because it can completely remove small breast lesions, it provides continuous and sufficient tissue specimens for pathological diagnosis through a single operation, while minimizing the damage to the breast tissue and maintaining the breast. The shape of the tissue is therefore widely used in the early diagnosis of breast cancer. Prognostic factors for recurrence of ipsilateral breast tumors after breast-sparing breast cancer include patient factors (young and BRCA gene mutations), tumor factors (large tumor burden, high histological grade, triple negative, and HER2 positive, etc.), and treatment factors (did not receive adjuvant radiotherapy and systemic treatment), and the resection of benign breast lesions. Due to the fluctuating accuracy of fine-needle aspiration (FNA) biopsy and the inability to distinguish between carcinoma in situ and invasion, it is no longer routinely used in most hospitals. Currently, the most commonly used biopsy methods in breast surgery are hollow needle aspiration biopsy (CNB) and vacuum-assisted biopsy (VAB) (3). VAB is accurate, simple and has fewer complications. According to the existing literature, the sensitivity of VAB is $85-97 \%$ and the false negative rate is $0-9 \%(4,5)$. With the rapid development of medical standards, doctors recommend that women have mammograms. Mammography can reduce the death rate from breast cancer (6). However, varying degrees of lesions in thymus tissue have been detected during screening, which could not be distinguished from malignancy, such that a histological evaluation was required (7). Approximately $20 \%$ of suspicious lesions have been found to be malignant upon histological evaluation $(8,9)$. In addition, future imaging may be compromised by scarring after a surgical biopsy. Minimally invasive surgery is an ideal option. Recent data on the accuracy of fine needle aspiration biopsy (FNAB) vary widely. Ductal carcinoma in situ (DCIS) or invasive carcinoma (IC) cannot be distinguished with FNAB (10). Core needle biopsy
(CNB) achieved $85-97 \%$ sensitivity and 100\% specificity $(11,12)$. However, the high correlation between imaging and histological findings is compelling. Inconsistencies between mammography and histological assessment require repeat $\mathrm{CNB}$ or open surgery $(13,14)$. One limitation of microcalcification assessment using $\mathrm{CNB}$ is that insufficient samples may be obtained. Therefore, CNB failed to improve calcification surgery in patients compared to highquality surgery $(15,16)$. Proposed in 1995 to address these problems $(17,18)$, vacuum-assisted breast biopsy (VAB) has the following characteristics: single insertion, the ability to obtain consecutive and larger tissue samples, and the ability to orient samples. The biopsy instrument is 11 inches in diameter and allows more samples to be obtained. The tissue volume of VAB is 10 times larger than that of CNB $(19,20)$. Therefore, VAB is more accurate than CNB in assessing microcalcifications. Much research $(19,20)$ has been conducted on the usefulness of VAB in the early diagnosis of breast tumors. It can lead to more accurate conclusions to provide clinical guidance.

The purpose of this systematic review was to summarize the evidence on case detection rates and assess the results of the diagnosis and treatment value of ultrasound-guided $\mathrm{VAB}$ for breast lesions. A total of 10 articles were included, the results of domestic and foreign comparative studies were reviewed, and a meta-analysis was performed. This meta-analysis provides the scientific and theoretical basis to enhance the accuracy of breast cancer biopsy detection, improve the detection rate of breast cancer and reduce detection sequelae.

We present the following article in accordance with the PRISMA reporting checklist (available at https://dx.doi. org/10.21037/gs-21-611).

\section{Methods}

\section{Literature search strategy}

PubMed, Medline, Embase, and Cochrane Central Register of Controlled Trials database were searched, related research published from January 1, 2001 to March 2021 was analyzed. The search keywords included breast tumors, breast cancer related diseases, breast lesions, vacuumassisted breast biopsy, sensitivity and specificity, safety, accuracy, missed diagnosis rate, underestimation rate and other descriptive terms. According to the pre-established inclusion and exclusion criteria, the full text of the target document was obtained, manual search was conducted, and 
the required documents were screened.

\section{Inclusion and exclusion criteria}

Inclusion criteria: (I) documents containing keywords; (II) patients experienced preterm labor and premature rupture of membranes; (III) clinical trials; (IV) the patient had breast cancer; (V) patients aged 18 years and over; (VI) articles had to be published, and non-published articles were not considered.

Exclusion criteria: (I) duplicate publications; (II) articles that did not report the outcomes being assessed; (III) nonclinical trials; (IV) other methods of biopsy; (V) patients with other breast diseases; (VI) articles in Chinese language; (VII) other meta-analyses were used as references but not included in the analyses; and/or (VIII) unclear results and incomplete patient data records.

\section{Literature screening}

Articles were screened independently and results were compared. If there was a difference, it needed to consult a breast lesion specialist in the hospital for an assessment of the difference.

\section{Data extraction}

Data extraction in this study was carried out independently by two researchers. During data extraction, data were extracted independently by two researchers, and Excel tables were used to capture basic details of the articles, characteristics of the research objects, intervention measures, outcome indicators, and risk of bias evaluations. After extraction, crosschecks were conducted. During the process of data extraction, if there were differences of opinions, they were discussed and resolved through consultation with a third researcher. The following data were extracted: basic article information (title, author, year of publication, author), basic characteristics of subjects (gender, age, study sample size, baseline comparability), the method of literature research, research design, experimental and control group interventions, and research result.

\section{Quality assessment}

Risk of bias of the included articles was assessed using criteria specified in the Cochrane Handbook for Systematic Reviews of Intervention 5.0.2. Specifically, it included: (I) generation of random sequence (whether a random number table or other random method was utilized for grouping of research objects); (II) allocation concealment (whether there was randomization and whether the randomization remained concealed); (III) subject blinding (whether study subjects knew whether they were in the experimental group and which group they were in); (IV) outcome assessor blinding (whether the researcher or outcome assessor knew the group status of subjects); (V) data integrity (whether data were complete and whether there were missing data); (VI) selective reporting (whether there was selective reporting); and (VII) other biases. If data extracted by the two researchers were inconsistent, the inconsistency was discussed and resolved. If no consensus was achieved, a third researcher was consulted.

\section{Result display}

Forest plots clearly show the results of individual studies and combine studies with corresponding confidence intervals. If there is no overlap between the confidence intervals of the individual studies, the plot indicates statistical inhomogeneity between the studies. Further subgroup analysis is required to combine stochastic and fixed models with acceptable inhomogeneity. Subgroups are divided according to different study designs, and then the impact of each subgroup can be ignored if the inhomogeneity between studies is substantial and the sources of the inhomogeneity cannot be addressed. An appropriate statistical model was selected. Sensitivity analysis: Sensitivity analysis of the research results was performed by investigating whether individual studies affected the overall results of the included studies substantially. Each study included in the meta-analysis was removed one at a time. Combined with the results of the remaining studies, the combined results of each study were compared with the individual results to confirm whether the results were the same. Generally, individual studies will influence the overall results substantially under the following two circumstances. First, if a study is deleted, the presumption of the size of the combined effect is $95 \%$ of the size of the combined effect. When a study is deleted, the results yield significantly different results. If one study affects the overall results with little difference, it indicates the sensitivity of the combined results and the results obtained are not stable. On the contrary, in this case, the results show that the sensitivity is stable and the conclusions are correct. 


\section{Statistical analysis}

RevMan5.3 software provided by the Cochrane Collaboration was used for meta-analysis. Odds ratios (OR) were used describe the effect size, together with $95 \%$ confidence interval (CI). Heterogeneity was first tested for the included studies, with $\alpha=0.1$ as the test level. If there was no heterogeneity among the studies $(\mathrm{P}>0.1$, $\mathrm{I}^{2}<50 \%$ ), a fixed-effect model was selected for metaanalysis; otherwise, subgroup analysis was performed for the included data. $\mathrm{P}<0.05$ was considered as indicating statistical significance. When the number of references included in the analysis of a single risk factor was more than 10, funnel plots were used to analyze the publication bias of each risk factor. RevMan5.3 software was used to analyze publication bias using Begg's and Egger's tests. If the result yielded $\mathrm{P}>0.05$, it was considered that there was no publication bias, otherwise, it was considered that there was publication bias.

\section{Results}

\section{Literature search results}

A total of 2,519 literatures were included in the database from its construction to March 2021, and 1298 literatures were excluded from the retrieved articles. Based on the titles and abstracts read, 832 articles were excluded. Reading the full text, 89 cases of non-breast lesions were excluded, 211 cases of non-vacuum-assisted biopsy were excluded, and 82 cases were excluded that could not be combined with other RTC. Finally, 10 articles meeting the inclusion criteria were included in the analysis (21-30) (Figure 1, Tables 1-3).

\section{Bias-risk assessment of included articles}

The risk of bias of the 10 articles included in this study was judged using the Cocbrane Handbook (version 5.0.2) of the systematic review writing manual. Review Manager 5.3 was utilized to generate a chart illustrating the details of risk of bias (Figures 2,3).

\section{Meta-analysis of vacuum-assisted detection of breast cancer}

Figure 4 shows a forest plot illustrating sensitivity and specificity of diagnosis of breast cancer using VAB. As presented in Figure 4, the sensitivity ranged from 0.94 to 1.00 (mean, 0.981; 95\% CI: 0.972-0.987). In addition, specificity was $0.87-1.00$ (mean, 0.999 ; $95 \%$ CI: $0.997-$ 0.999). Sensitivity and specificity of $\chi^{2}$ were $37.10(\mathrm{P}=0.011)$ and $32.00(\mathrm{P}=0.043)$, respectively, and those of PLR, NLR, and DOR were $46.98(\mathrm{P}=0.001), 54.92(\mathrm{P}=0.001)$, and 43.49 $(\mathrm{P}=0.002)$, respectively, indicating notable heterogeneity between studies (Figure 4). In addition, PLR was 93.84 (95\% CI: 41.55-211.95), NLR was 0.05 (95\% CI: 0.03-0.09), and DOR was 1,891.7 (95\% CI: 683.8-5,233.4).

\section{High vacuum-assisted detection of breast cancer SROC curve}

The SROC curve provides a global summary of test performance and shows the trade-off between sensitivity and specificity. SROC plots of VAB biopsy results show true and false positive rates for individual studies. In this study, the SROC curve was near the ideal upper left corner of the SROC curve. The area under the curve (AUC) was 0.98, suggesting high accuracy (Figure 5). The maximum joint sensitivity and specificity $(q)$ was 0.93 .

\section{Discussion}

In this meta-analysis, a total of 10 articles (21-30) were included. Regarding the quality evaluations of the 10 articles, there was only one article with quality grade A and 9 articles with quality grade B. A total of 9 of the 10 studies reported basic data such as age, disease type, and disease stage. Limitations of the interventions in this study mean that measurement biases may be present. To improve the reliability and applicability of similar future research, the research methods and design need to be amended. Due to the large fluctuations in the accuracy of fine needle aspiration biopsy (FNAC) and the inability to distinguish between carcinoma in situ and invasiveness, most hospitals are no longer routinely used. At present, the most commonly used biopsy method in breast surgery is core needle aspiration biopsy $(\mathrm{CNB})$ and vacuum assisted biopsy (VAB) $(31,32)$. Compared with other methods, the biggest advantage of ultrasound-guided vacuum-assisted biopsy is its accuracy, simplicity, and fewer complications. The advantages of vacuum-assisted biopsy are precise positioning and accurate puncture of the lesion, especially for deeper and smaller tumors that are not clinically accessible. It also has sufficient materials in disease examination and immunohistochemical detection, it may provide more pathological information before treatment, which can greatly increase the detection rate of breast cancer and provide a basis for the formulation of clinical treatment plans (33). In addition, the puncture port is 


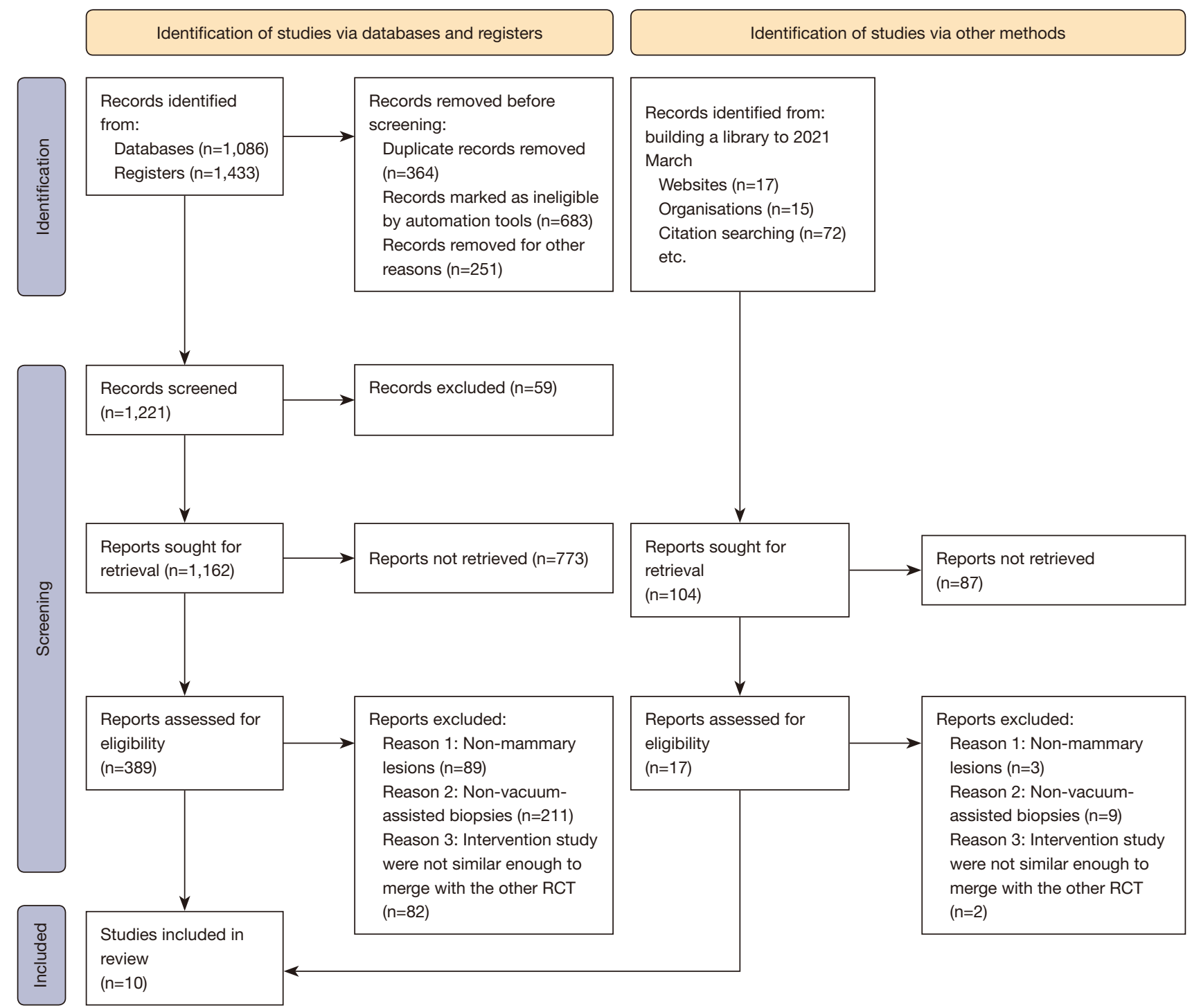

Figure 1 Literature retrieval process.

Table 1 Quality of 10 included studies

\begin{tabular}{lccccccccccccccc}
\hline & \multicolumn{1}{c}{ Quality Assessment of Diagnostic Accuracy Studies (QUADAS item) } \\
\cline { 2 - 12 } & 1 & 2 & 3 & 4 & 5 & 6 & 7 & 8 & 9 & 10 & 11 & 12 & 13 & 14 \\
\hline Yes (n) & 11 & 13 & 13 & 2 & 13 & 2 & 13 & 13 & 13 & 13 & 3 & 13 & 4 & 4 \\
No (n) & 3 & 0 & 0 & 11 & 13 & 11 & 0 & 0 & 0 & 0 & 0 & 0 & 0 & 0 \\
Unknown (n) & 0 & 0 & 0 & 0 & 0 & 0 & 0 & 0 & 0 & 0 & 10 & 0 & 12 & 10 \\
Yes (\%) & 86 & 100 & 100 & 10 & 0 & 86 & 100 & 100 & 100 & 100 & 5 & 100 & 10 & 10 \\
\hline
\end{tabular}


Table 2 Basic characteristics of included articles

\begin{tabular}{|c|c|c|c|c|c|}
\hline First author & Year & No. of cases & Biopsy needle size & Ultrasonic guidance or not & Range of age (years) \\
\hline Choo (22) & 2008 & 58 & 8 & Yes & 53 \\
\hline Hung (23) & 2001 & 49 & 11 & Yes & $22-58$ \\
\hline Kim (24) & 2008 & 59 & 11 & Yes & $20-70$ \\
\hline Meloni (26) & 2001 & 73 & 11 & Yes & $30-77$ \\
\hline Perretta (27) & 2008 & 47 & 9 & Yes & $30-73$ \\
\hline Shin (28) & 2008 & 123 & 8 & Yes & $21-75$ \\
\hline Simon (29) & 2000 & 67 & 11 & Yes & $23-82$ \\
\hline
\end{tabular}

Table 3 General information included in articles

\begin{tabular}{|c|c|c|c|c|c|c|c|}
\hline The first author & Year & No. of cases & TP & $\mathrm{FP}$ & $\mathrm{FN}$ & $\mathrm{TN}$ & Score \\
\hline Choo (22) & 2008 & 58 & 8 & 0 & 0 & 50 & 7 \\
\hline Hung (23) & 2001 & 49 & 4 & 0 & 0 & 45 & 7 \\
\hline Kim (24) & 2008 & 59 & 29 & 0 & 1 & 29 & 9 \\
\hline Meloni (26) & 2001 & 73 & 36 & 0 & 2 & 35 & 5 \\
\hline Perretta (27) & 2008 & 47 & 15 & 0 & 1 & 31 & 8 \\
\hline Shin (28) & 2008 & 123 & 2 & 0 & 0 & 121 & 9 \\
\hline Simon (29) & 2000 & 67 & 17 & 2 & 1 & 47 & 8 \\
\hline
\end{tabular}

TP, true positive; FP, false positive; FN, false negative; TN, true negative.

small and the cosmetic effect is good, only $3-5 \mathrm{~mm}$, no suture, no scar; and multiple lesions on the same breast can be punctured through one puncture port (less than 3, the distance is not more than $10 \mathrm{~cm}$ ). It avoids incision of the skin, subcutaneous tissue and normal glands. The tissue damage is small and the recovery is quick. For patients with deep breast masses and obesity, the advantages are particularly obvious (34). It can obtain larger specimens and completely solve the problem of insufficient specimen size, and as such diagnostic accuracy is higher. Due to the larger diameter of the excised specimen, it is a convenient and accurate approach for pathologists to use to detect prognostic indicators. This is particularly important for patients with locally advanced breast cancers, as these patients often show changes or loss of molecular information after neoadjuvant chemotherapy (35). At the same time, VAB can complete the diagnosis and treatment of benign tumors simultaneously and has characteristics similar to those of minimally invasive and aesthetic procedures. The disadvantage of $\mathrm{VAB}$ is that it is expensive and therefore may not be affordable for patients with limited financial means. Underestimation is present if a carcinomatous lesion is reported as high risk using $\mathrm{CNB}$ or $\mathrm{VAB}$, or if it is reported as ductal carcinoma in situ (DCIS) of the breast and confirmed as invasive cancer after surgery. Such high-risk lesions include atypical hyperplasia, lobular 
carcinoma in situ, radial scar, etc. Therefore, patients diagnosed as having high-risk lesions using the above two methods must undergo surgical biopsy to avoid missed diagnoses. Since the SROC curve is not easy to interpret and use in clinical practice, the value of likelihood ratio is more meaningful in clinical treatment and diagnosis, and PLR and NLR are used as indicators of diagnostic accuracy. Likelihood ratio is a method that combines the sensitivity and specificity of the test and assesses the probability of whether a positive or negative result alters an existing condition, such as disease status. The PLR was 93.8 , indicating that patients with breast cancer were about 94 times more likely to have a positive VAB result than those without breast cancer. This high probability

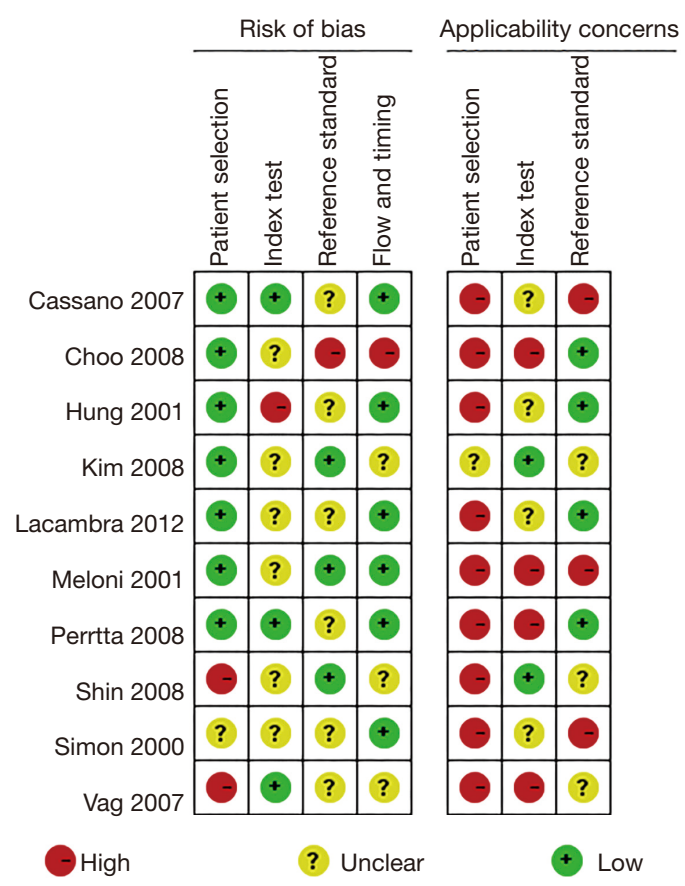

Figure 2 Risk of bias assessment diagram of included articles. suggests that a positive $\mathrm{VAB}$ result can initiate surgery or other treatment. NLR was found to be 0.05 . Overall, the quality of the included studies was higher than the median level of Quality Assessment of Diagnostic Accuracy Studies (QUADAS). Some studies failed to meet item 4 (disease progression bias), 5 (partial validation), 11 (reference standard review bias), 13 (unexplained test results), or 14 (withdrawal). Based on detailed analysis of the QUADAS assessment tool and the included studies, there may be erroneous classification biases. With the exception of two articles $(28,31)$, most studies conducted follow up for less than 2 years. These biases can affect the accuracy of the $\mathrm{VAB}$ analysis. Various aspects were included in the analysis of the basic data: QUADAS score, needle size, imaging guidance system, patient testing location, and methodology and design (prospective and retrospective studies).

In this meta-analysis, a lot of foreign studies were included, which addressed various risk factors. In addition, since there were no comparisons between the control and treatment groups, the relevant Chinese literature was not included. In addition, the treatment time of 10 included studies in this study was contradictory, which may have influenced the results of this meta-analysis. Randomization methods were not reported. Therefore, it is recommended to further improve experimental plans; standardize the specific duration of follow up, methodology and drug of the intervention; and implement high-quality, large-scale sample, multi-center randomized controlled trials to obtain more reliable evidence.

\section{Conclusions}

To summarize the evidence of the case detection rate and study the results of the diagnosis and treatment value of ultrasound-guided VAB for breast lesions, 10 articles were included, and the results of domestic and foreign studies were reviewed, and various screening and meta-analysis

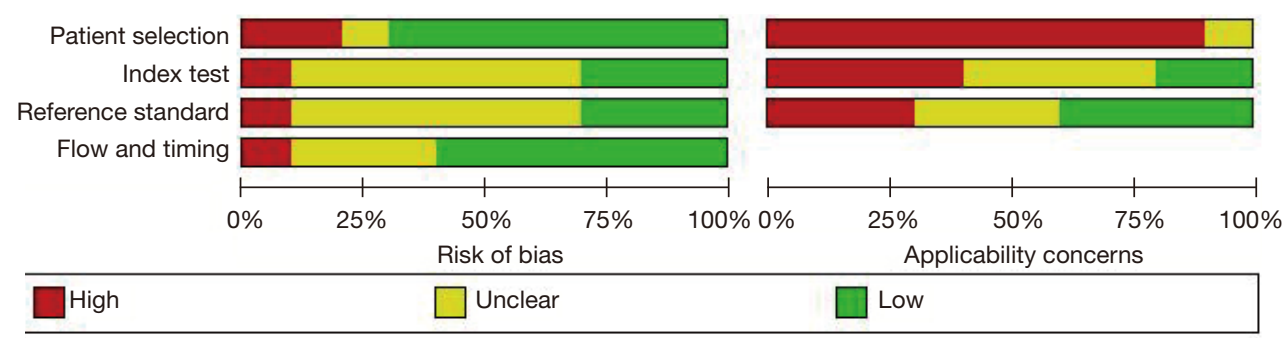

Figure 3 Risk of bias evaluation bar graph of included articles. 


Study
Cassano 2007
Choo 2008
Hung 2001
Kim 2008
Lacambra 2012
Meloni 2001
Perretta 2008
Shin 2008
Simon 2000
Vag 2007

$\begin{array}{rrrrr}\text { TP } & \text { FP } & \text { FN } & \text { TN } & \text { Sensitivity }(95 \% \text { Cl) } \\ 76 & 0 & 2 & 188 & 0.97[0.91,1.00] \\ 8 & 0 & 0 & 50 & 1.00[0.63,1.00] \\ 4 & 0 & 0 & 45 & 1.00[0.40,1.00] \\ 29 & 0 & 1 & 29 & 0.97[0.83,1.00] \\ 0 & 0 & 75 & 10 & 0.00[0.00,0.05] \\ 36 & 0 & 2 & 35 & 0.95[0.82,0.99] \\ 15 & 0 & 1 & 31 & 0.94[0.70,1.00] \\ 2 & 0 & 0 & 121 & 1.00[0.16,1.00] \\ 17 & 2 & 1 & 47 & 0.94[0.73,1.00] \\ 28 & 0 & 1 & 36 & 0.97[0.82,1.00]\end{array}$

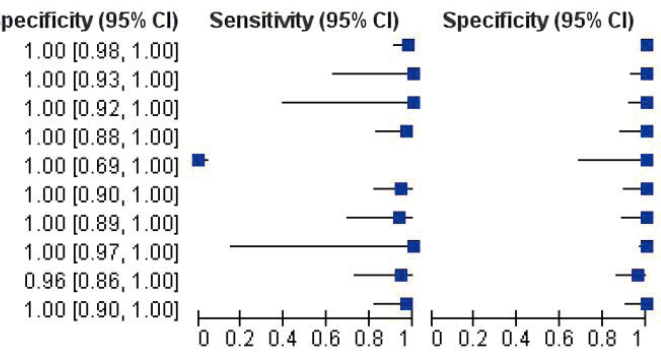

Figure 4 Forest plot of sensitivity and specificity to vacuum-assisted biopsy in breast cancer diagnosis.

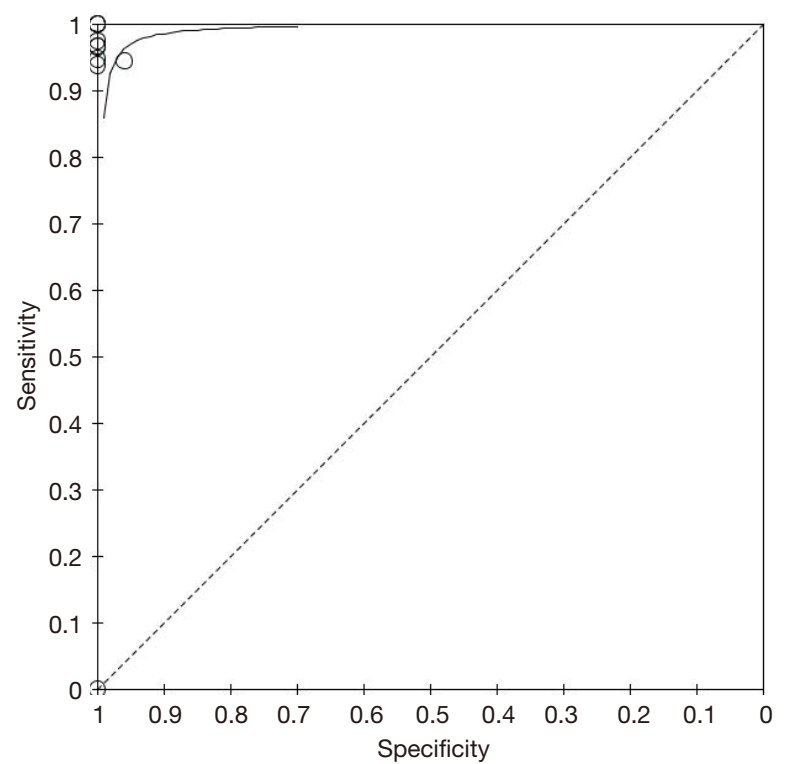

Figure 5 Summary receiver operating characteristic (SROC) curve of vacuum-assisted biopsy.

were conducted. VAB has proven to be an accurate biopsy method to detect breast cancer in women. The results of the meta-analysis were stable, and VAB showed high sensitivity (98\%) and specificity $(100 \%)$, indicating that it is a promising alternative to open breast biopsy and can improve breast cancer therapy. However, this meta-analysis has some limitations: the retrieval of articles may not have been sufficiently comprehensive; and there were differences in risk factor assessment and measurement in different studies, which may have affected the results. All the studies included in the meta-analysis were published articles, and the failure to include unpublished sources may have led to potential publication bias. It is suggested to carry out more high-quality and multicenter original studies with large sample sizes for verification of results in the future to provide clinical guidance and a scientific basis for the treatment of breast cancer and more rapid and efficient detection of breast cancer.

\section{Acknowledgments}

Funding: None.

\section{Footnote}

Reporting Checklist: The authors have completed the PRISMA reporting checklist. Available at https://dx.doi. org/10.21037/gs-21-611

Conflicts of Interest: All authors have completed the ICMJE uniform disclosure form (available at https://dx.doi. org/10.21037/gs-21-611). The authors have no conflicts of interest to declare.

Ethical Statement: The authors are accountable fsor all aspects of the work in ensuring that questions related to the accuracy or integrity of any part of the work are appropriately investigated and resolved.

Open Access Statement: This is an Open Access article distributed in accordance with the Creative Commons Attribution-NonCommercial-NoDerivs 4.0 International License (CC BY-NC-ND 4.0), which permits the noncommercial replication and distribution of the article with the strict proviso that no changes or edits are made and the original work is properly cited (including links to both the formal publication through the relevant DOI and the license). See: https://creativecommons.org/licenses/by-nc-nd/4.0/.

\section{References}

1. Bray F, Ferlay J, Soerjomataram I, et al. Global cancer 
statistics 2018: GLOBOCAN estimates of incidence and mortality worldwide for 36 cancers in 185 countries. CA Cancer J Clin 2018;68:394-424.

2. Wang H, Mao X. Evaluation of the Efficacy of Neoadjuvant Chemotherapy for Breast Cancer. Drug Des Devel Ther 2020;14:2423-33.

3. Li X, Gao H, Xu M, et al. Breast papillary lesions diagnosed and treated using ultrasound-guided vacuumassisted excision. BMC Surg 2020;20:204.

4. Pijnappel RM, van den Donk M, Holland R, et al. Diagnostic accuracy for different strategies of imageguided breast intervention in cases of nonpalpable breast lesions. Br J Cancer 2004;90:595-600.

5. Gøtzsche PC, Olsen O. Is screening for breast cancer with mammography justifiable? Lancet 2000;355:129-34.

6. Olsen O, Gøtzsche PC. Cochrane review on screening for breast cancer with mammography. Lancet 2001;358:1340-2.

7. Elmore JG, Barton MB, Moceri VM, et al. Ten-year risk of false positive screening mammograms and clinical breast examinations. N Engl J Med 1998;338:1089-96.

8. Kopans DB. The positive predictive value of mammography. AJR Am J Roentgenol 1992;158:521-6.

9. Brett J, Austoker J, Ong G. Do women who undergo further investigation for breast screening suffer adverse psychological consequences? A multi-centre follow-up study comparing different breast screening result groups five months after their last breast screening appointment. J Public Health Med 1998;20:396-403.

10. Lidbrink E, Elfving J, Frisell J, et al. Neglected aspects of false positive findings of mammography in breast cancer screening: analysis of false positive cases from the Stockholm trial. BMJ 1996;312:273-6.

11. Lowe JB, Balanda KP, Del Mar C, et al. Psychologic distress in women with abnormal findings in mass mammography screening. Cancer 1999;85:1114-8.

12. Pisano ED, Fajardo LL, Tsimikas J, et al. Rate of insufficient samples for fine-needle aspiration for nonpalpable breast lesions in a multicenter clinical trial: The Radiologic Diagnostic Oncology Group 5 Study. The RDOG5 investigators. Cancer 1998;82:679-88.

13. Ciatto S, Brancato B, Risso G, et al. Accuracy of fine needle aspiration cytology (FNAC) of axillary lymph nodes as a triage test in breast cancer staging. Breast Cancer Res Treat 2007;103:85-91.

14. Mueller-Holzner E, Frede T, Daniaux M, et al. Ultrasound-guided core needle biopsy of the breast: does frozen section give an accurate diagnosis? Breast Cancer
Res Treat 2007;106:399-406.

15. Verkooijen HM, Peeters PH, Buskens E, et al. Diagnostic accuracy of large-core needle biopsy for nonpalpable breast disease: a meta-analysis. Br J Cancer 2000;82:1017-21.

16. Verkooijen HM, Borel Rinkes IH, Peeters PH, et al. Impact of stereotactic large-core needle biopsy on diagnosis and surgical treatment of nonpalpable breast cancer. Eur J Surg Oncol 2001;27:244-9.

17. Liberman L, Dershaw DD, Glassman JR, et al. Analysis of cancers not diagnosed at stereotactic core breast biopsy. Radiology 1997;203:151-7.

18. Brenner RJ, Fajardo L, Fisher PR, et al. Percutaneous core biopsy of the breast: effect of operator experience and number of samples on diagnostic accuracy. AJR Am J Roentgenol 1996;166:341-6.

19. Parker SH, Burbank F. A practical approach to minimally invasive breast biopsy. Radiology 1996;200:11-20.

20. Burbank F, Parker SH, Fogarty TJ. Stereotactic breast biopsy: improved tissue harvesting with the Mammotome. Am Surg 1996;62:738-44.

21. Cassano E, Urban LA, Pizzamiglio M, et al. Ultrasoundguided vacuum-assisted core breast biopsy: experience with 406 cases. Breast Cancer Res Treat 2007;102:103-10.

22. Choo KS, Kwak HS, Tae Bae Y, et al. The value of a combination of wire localization and ultrasoundguided vacuum-assisted breast biopsy for clustered microcalcifications. Breast 2008;17:611-6.

23. Hung WK, Lam HS, Lau Y, et al. Diagnostic accuracy of vacuum-assisted biopsy device for image-detected breast lesions. ANZ J Surg 2001;71:457-60.

24. Kim HS, Kim MJ, Kim EK, et al. US-guided vacuumassisted biopsy of microcalcifications in breast lesions and long-term follow-up results. Korean J Radiol 2008;9:503-9.

25. Lacambra MD, Lam CC, Mendoza P, et al. Biopsy sampling of breast lesions: comparison of core needle- and vacuum-assisted breast biopsies. Breast Cancer Res Treat 2012;132:917-23.

26. Meloni GB, Dessole S, Becchere MP, et al. Ultrasoundguided mammotome vacuum biopsy for the diagnosis of impalpable breast lesions. Ultrasound Obstet Gynecol 2001;18:520-4.

27. Perretta T, Pistolese CA, Bolacchi F, et al. MR imagingguided 10-gauge vacuum-assisted breast biopsy: histological characterisation. Radiol Med 2008;113:830-40.

28. Shin HJ, Kim HH, Kim SM, et al. Papillary lesions of the 
breast diagnosed at percutaneous sonographically guided biopsy: comparison of sonographic features and biopsy methods. AJR Am J Roentgenol 2008;190:630-6.

29. Simon JR, Kalbhen CL, Cooper RA, et al. Accuracy and complication rates of US-guided vacuum-assisted core breast biopsy: initial results. Radiology 2000;215:694-7.

30. Vag T, Pfleiderer SO, Böttcher J, et al. Ultrasound-guided breast biopsy using a 10-gauge self-contained vacuumassisted device. Eur Radiol 2007;17:3100-2.

31. Hoffmann J, Marx M, Hengstmann A, et al. UltrasoundAssisted Tumor Surgery in Breast Cancer - A Prospective, Randomized, Single-Center Study (MAC 001). Ultraschall Med 2019;40:326-32.

32. Pei L, Zhou Y, Tan G, et al. Ultrasound-Assisted Thoracic Paravertebral Block Reduces Intraoperative Opioid

Cite this article as: $\mathrm{Lu}$ W, Tu L, Xie D, Yao F, Lin L, Li Y, Li D, Mou C. A systematic review and meta-analysis: value of ultrasound-guided vacuum-assisted biopsy in the diagnosis and treatment of breast lesions. Gland Surg 2021;10(10):3020-3029. doi: 10.21037/gs-21-611
Requirement and Improves Analgesia after Breast Cancer Surgery: A Randomized, Controlled, Single-Center Trial. PLoS One 2015;10:e0142249.

33. Zandi A, Khayamian MA, Saghafi M, et al. MicroneedleBased Generation of Microbubbles in Cancer Tumors to Improve Ultrasound-Assisted Drug Delivery. Adv Healthc Mater 2019;8:e1900613.

34. Sim LS, Kei PL. Upright stereotactic vacuum-assisted needle biopsy of suspicious breast microcalcifications. J Med Imaging Radiat Oncol 2008;52:358-64.

35. Montemurro F, Nuzzolese I, Ponzone R. Neoadjuvant or adjuvant chemotherapy in early breast cancer? Expert Opin Pharmacother 2020;21:1071-82.

(English Language Editor: B. Meiser) 